\title{
The association between different molecular weights of hyaluronic acid and CHAD, HIF-1 $\alpha$, COL2A1 expression in chondrocyte cultures
}

\author{
DUYGU YASAR SIRIN ${ }^{1}$, NECATI KAPLAN ${ }^{2}$, IBRAHIM YILMAZ $^{3}$, NUMAN KARAARSLAN $^{4}$, \\ HANEFI OZBEK ${ }^{3}$, YENER AKYUVA ${ }^{5}$, YASIN EMRE KAYA ${ }^{6}$, KADIR OZNAM ${ }^{7}$, NURAY AKKAYA ${ }^{8}$, \\ OLCAY GULER $^{9}$, SEMIH AKKAYA ${ }^{10}$ and MAHIR MAHIROGULLARI ${ }^{11}$
}

\footnotetext{
${ }^{1}$ Department of Molecular Biology and Genetics, Faculty of Arts and Sciences, Namik Kemal University, Tekirdag 59030;

${ }^{2}$ Department of Neurosurgery, Istanbul Rumeli University, Tekirdag 59680; ${ }^{3}$ Department of Medical Pharmacology, Istanbul Medipol University School of Medicine, Istanbul 34810; ${ }^{4}$ Department of Neurosurgery, Namik Kemal University School of Medicine, Tekirdag 59060; ${ }^{5}$ Department of Neurosurgery, Gaziosmanpasa Taksim Training and Research Hospital,

Istanbul 34433; ${ }^{6}$ Department of Orthopedics and Traumatology, Abant Izzet Baysal University School of Medicine,

Bolu 14000; ${ }^{7}$ Department of Orthopedics and Traumatology, Istanbul Medipol University School of Medicine,

Istanbul 34214; ${ }^{8}$ Department of Physical Medicine and Rehabilitation, Pamukkale University School of Medicine, Denizli 20070; ${ }^{9}$ Department of Orthopedics and Traumatology, Medical Park Health Group, Istanbul 34180;

${ }^{10}$ Department of Orthopedics and Traumatology, Denizli Private Hospital, Denizli 20070; ${ }^{11}$ Department of Orthopedics and Traumatology, Memorial Health Group, Istanbul 34758, Turkey
}

Received October 5, 2017; Accepted January 10, 2018

DOI: $10.3892 /$ etm.2018.5943

\begin{abstract}
The aim of the present study was to investigate the effects of three different formulations of hyaluronic acid (HA): Low molecular weight (MW) Sinovial One ${ }^{\circledR}$, medium MW Viscoplus ${ }^{\circledR}$ and high MW Durolane ${ }^{\circledR}$, on chondrocyte proliferation and collagen type II (COL2A1), hypoxia-inducible factor $1 \alpha(\mathrm{HIF}-1 \alpha)$ and chondroadherin (CHAD) expression in primary chondrocyte cultures. Standard primary chondrocyte cultures were established from osteochondral tissues surgically obtained from 6 patients with gonarthrosis. Cell morphology was evaluated using an inverted light microscope; cell proliferation was determined with a MTT assay and confirmed with acridine orange/propidium iodide staining. Levels of CHAD, COL2A1 and HIF-1 $\alpha$ expression were assessed using specific TaqMan gene expression assays. The results demonstrated the positive effect of HA treatment on cell proliferation, which
\end{abstract}

Correspondence to: Dr Duygu Yasar Sirin, Department of Molecular Biology and Genetics, Faculty of Arts and Sciences, Namik Kemal University, 1 Campus Street, Tekirdag 59030, Turkey E-mail: dysirin@nku.edu.tr

Abbreviations: CHAD, chondroadherin; COL2A1, collagen type II $\alpha 1$ chain; ECM, extracellular matrix; HA, hyaluronic acid; HIF-1 $\alpha$, hypoxia-inducible factor $1 \alpha$; MW, molecular weight; OA, osteoarthritis; RQ, relative quantity

Key words: chondroadherin, collagen type II, hypoxia-inducible factor $1 \alpha$, hyaluronic acid, chondrocytes was independent from the MW. COL2A1 expression increased in the medium and high MW HA treated groups. It was observed that HIF-1 $\alpha$ expression increased in the high MW treated group alone. CHAD expression increased only in the medium MW HA treated group. Evaluation of gene expression revealed that levels of expression increased as the duration of HA application increased, in the medium and high MW HA treated groups. In terms of increased viability and proliferation, a longer duration of HA application was more effective. Taken together, it may be concluded that the administration of medium and high MW HA may be a successful way of treating diseases affecting chondrocytes in a clinical setting.

\section{Introduction}

Osteoarthritis (OA) is the most common form of arthritis and a major cause of reduced activity and disability, with an increased incidence after 65 years of age, which is characterized by progressive cartilage degradation (1-3). The primary aims of treatments for OA are to reduce the pain and disability experienced by patients, thus improving quality of life. Various medications, including analgesics, non-steroidal anti-inflammatories, platelet-rich plasma and steroids, are used in combination with physical therapy and/or surgery to treat those with OA (4). However, novel treatments to prevent chondrocyte degeneration are required, as current treatments only alleviate symptoms of OA and do not tackle the underlying causes of the disease (5). In addition to analgesics, non-steroidal anti-inflammatories, platelet-rich plasma and steroids, intra-articular injections of hyaluronic acid (HA), a slow-acting chondroprotective agent, are frequently administered to 
patients with OA $(5,6)$. HA has a more prolonged effect and avoids several of the side effects associated with conventional OA treatment, including gastrointestinal toxicity or increased risk of the cardiovascular events $(4,7,8)$.

$\mathrm{HA}$ is a long chain polysaccharide present in loose connective tissue, skin, the eyes and synovial fluid $(4,7,9)$. HA is concentrated at the surface of the articular cartilage and the superficial layers of the synovial membrane and forms a semi-permeable barrier between the synovial fluid and cartilage. Marked decreases in HA concentration is associated with the onset of OA (4,7). In pathological conditions, the molecular weight (MW) of HA also decreases, which increases the cartilage mechanical load, thus inducing damage of the cartilage $(1,9)$. The administration of HA was previously determined to reduce chondrocyte apoptosis rate in patients with OA (10). The role of HA in OA remains unclear; however, it may promote cell proliferation and pericellular matrix formation $(4,7,11)$.

In the present study, the effect of different MWs of HA on the proliferation of chondrocytes, as well as the expression of collagen type II (COL2A1), hypoxia-inducible factor $1 \alpha$ (HIF-1 $\alpha$ ) and chondroadherin (CHAD) in chondrocytes was investigated. To the best of our knowledge, the current study is the first to determine the effects of HA on the expression of HIF-1 and CHAD. Impairment of anabolic and catabolic processes is common features of OA. In healthy cartilage, the presence of COL2A1 is a marker of anabolism (5). The COL2Al gene encodes an important cartilage-specific matrix protein and its expression is regulated by transcription factors, including SOX9 and transforming growth factor $B$ (12). Furthermore, it has been demonstrated that an increase in $C O L 2 A 1$ expression induces the production of extracellular matrix (ECM) (12).

HIF-1 regulates the transcription of a wide range of genes involved in glucose metabolism, angiogenesis and cell survival $(2,3)$. The HIF-1 heterodimeric transcription complex is composed of HIF- $1 \alpha$ and HIF-1 $\beta$ subunits. A hypoxic environment, hormones and growth factors can induce the translocation of HIF-1 to the nucleus, where it associates with its corresponding subunit to form an active HIF-1 transcription factor that may regulate the expression of its target genes $(2,3)$. HIF-1 $\alpha$ regulates hypoxia-induced ECM synthesis in chondrocytes, as well as cartilage regeneration (13-15).

CHAD is a class IV small leucine-rich proteoglycan/protein that mediates the signaling between chondrocytes and the ECM (16). CHAD binds to the $\alpha 2 \beta 1$ integrin, cell surface proteoglycans and type II collagen and serves a role in the regulation of chondrocyte signaling and cartilage homeostasis (16).

The present study investigated the effect of treatment with low, medium and high MW HA on COL2A1, HIF-1 $\alpha$ and CHAD expression in chondrocytes. Furthermore, the effect of HA on chondrocyte viability and proliferation was assessed. The aim of the current study was to determine whether the administration of different MWs of HA may be an effective method of treating patients with OA in a clinical setting.

\section{Materials and methods}

Patient selection. Cartilage tissue was obtained from 14 patients diagnosed with OA from Istanbul Medipol
University Hospital, Namik Kemal University Hospital, Gaziosmanpasa Taksim Training and Research Hospital, Abant Izzet Baysal University Hospital, Istanbul Medipol University Hospital, Pamukkale University Hospital, Medical Park Hospital, Denizli Private Hospital and Memorial Hospital. Osteophytes were graded using the Kellgren-Lawrence radiological grading scale (17). Patients were excluded if they had defects in kidney function or an allergy to a specific protein, including milk, egg or avian, or if they had taken methotrexate, fludarabine, cyclophosphamide, high doses of steroid and/or pressor substances, monoamine oxidase, coumarin group anticoagulants, phenytoin, primidone, phenylbutazone or tricyclic antidepressants in the preceding year. A total of 6 patients ( 3 female and 3 male; mean age, $64.92 \pm 3.68$ years) were included in the current study. These patients were also unresponsive to medical and conservative treatments for gonarthrosis. The present study was performed with the approval of the Local Ethics Board of Pamukkale University (Denizli, Turkey). Informed consent was obtained from patients attending the Orthopedics and Traumatology Clinic of all the aforementioned hospitals between August 2016 and June 2017, for use of their cells. Researchers did not know which groups received drugs and were blinded to which drugs were applied to the culture.

Study design and in vitro experimental setup. Standard primary chondrocyte cultures were established from surgically obtained osteochondral tissues taken from all 6 patients. Chondrocyte cultures were monitored using an inverted microscope. HAs of different MWs has been prepared using the appropriate solutions and concentrations. In the well plates, color-coded HAs were added and an extra plate from each treatment was set aside for MTT analysis, acridin orange/propidium iodide (AO/PI) staining and reverse transcription-quantitative polymerase chain reaction (RT-qPCR).

Isolation of primary chondrocytes. Osteochondral tissues were obtained from the distal femur and proximal tibia in patients with OA undergoing total knee arthroplasty for gonarthrosis. Tissues were transferred to the laboratory and cultured at $37^{\circ} \mathrm{C}$ in Dulbecco's modified Eagle's medium supplemented with $1 \%$ penicillin-streptomycin, $15 \%$ fetal bovine serum and 1\% L-glutamine (all Gibco; Thermo Fisher Scientific, Inc., Waltham, MA, USA). Osteochondral tissue samples were irrigated with $0.9 \%$ isotonic sodium chloride solution in a laminar flow cabinet to isolate samples from red blood cells. Tissues were dissected into $0.4 \mathrm{~cm}^{3}$ pieces, washed in Hank's balanced salt solution (Gibco; Thermo Fisher Scientific, Inc.) and transferred to Falcon tubes. Collagenase type II (0.375 mg; Gibco; Thermo Fisher Scientific, Inc.) dissolved in complete medium was added and incubated with tissues at $37^{\circ} \mathrm{C}$ overnight in $5 \%$ $\mathrm{CO}_{2}$. Samples were then centrifuged at a speed of $130 \mathrm{x} \mathrm{g}$ for $10 \mathrm{~min}$ in a coolant centrifuge at $4^{\circ} \mathrm{C}$. Cells were subsequently re-suspended in culture medium and transferred to $\mathrm{T} 75$ flasks prior to incubation at $37^{\circ} \mathrm{C}$ with $5 \% \mathrm{CO}_{2}$ to obtain primary cell cultures. The cultures were incubated until they became a monolayer confluent culture. Incubation duration differs between samples depending on the age of the patient, viable cell count, transport conditions and to doubling times of cells where they originated from, as previously described (18). 
Monolayer primary chondrocyte cultures were trypsinized and viable cells were identified via staining with trypan blue. Cell suspensions were seeded at a density of $1 \times 10^{4}$ cells/well in 96-well plates for MTT analysis, 3x10 4 cells/well in 24-well plates for $\mathrm{AO} / \mathrm{PI}$ analysis and $4 \times 10^{6}$ cell/dish in $100-\mathrm{mm}$ petri dishes for RNA isolation. Following $24 \mathrm{~h}$ incubation at $37^{\circ} \mathrm{C}$, low, medium and high MW HA was added to these cell cultures $(0 \mathrm{~h})$. Results from each experiment were obtained at 0,24 and $48 \mathrm{~h}$ following HA treatment.

Preparation and application of HA. The stock solution concentrations of the HA preparations used were: Low MW (800-1,200 kDa; Sinovial One ${ }^{\circledR} ; 50$ mg/2.5 ml; IBSA Farmaceutici, Lodi, Italia), medium MW (2,500 kDa; Viscoplus ${ }^{\circledR} ; 75 \mathrm{mg} / 3 \mathrm{ml}$; BioMedical B. Baumann $\mathrm{GmbH}$, Rodgau, Germany) and high MW HA (10,000 kDa; Durolane ${ }^{\circledR}$; $60 \mathrm{mg} / \mathrm{ml}$; Bioventus LCC, Durham, NC, USA). A HA concentration of $1 \mu \mathrm{M}$ was used in all experiments, as the majority of pharmaceutical formulations are administered at this concentration (19).

AO/PI staining. The AO/PI staining solution consisted of $10 \mathrm{~g}$ disodium-EDTA, $4 \mathrm{mg}$ PI, $50 \mathrm{ml} \mathrm{FBS}$ and $4 \mathrm{mg}$ AO (dissolved in $2 \mathrm{ml} 99 \%$ ethanol). The solution was mixed well and sterile distilled water was added to form a final volume of $200 \mathrm{ml}$. After incubation for $10 \mathrm{~min}$ at room temperature, cell cultures were monitored using a fluorescent microscope.

The nucleic acid binding dyes AO and PI may be used to accurately determine cell viability. AO is an intercalating dye able to permeate living and dead cells; it stains all nucleated cells and generates green fluorescence. PI can only enter dead cells with poor membrane integrity; it stains all dead nucleated cells to generate red fluorescence. Following staining with $\mathrm{AO} / \mathrm{PI}$, live nucleated cells exhibit green fluorescence and all dead nucleated cells exhibit red fluorescence (20).

Imaging with inverted and fluorescence microscopy. An inverted light microscope (magnification, x 20; CKX41; Olympus Corporation, Tokyo, Japan) was used to monitor cell cultures and a fluorescent microscope (magnification, x20; DM2500; Leica Microsystems, Inc., Buffalo Grove, IL, USA) was used for analysis of AO/PI staining. Microphotographs of the cell organizations were obtained prior to and during HA treatment and images were evaluated using the CytoVision capture station imaging program V.7.2 (Leica Microsystems, Inc.).

MTT analysis. Cell viability was assessed using a commercial MTT kit (Vybrant ${ }^{\circledR}$ MTT cell proliferation assay kit; Thermo Fisher Scientific, Inc.) following the manufacturer's protocol. MTT was prepared by adding $1 \mathrm{ml}$ sterile PBS to a vial containing $5 \mathrm{mg}$ MTT to obtain a $12 \mathrm{mM}$ MTT stock solution. To analyze cell viability, 10\% MTT solution prepared with complete medium was applied to monolayer cultures. Cultures were then incubated in a dark environment at $37^{\circ} \mathrm{C}$ for $4 \mathrm{~h}$. At the end of the incubation period, dimethyl sulfoxide was added to dissolve the purple formazan and incubated at $37^{\circ} \mathrm{C}$ for an additional $10 \mathrm{~min}$. Absorbance was then measured at a wavelength of $540 \mathrm{~nm}$. MTT analysis was performed prior to the addition of HA ( $0 \mathrm{~h}$; control) as well as 24 and $48 \mathrm{~h}$ following HA addition (18,20-25). Absorbance was measured using a microplate reader (Mindray MR-96A; Shenzhen Mindray Bio-Medical Electronics Co., Ltd., Shenzhen, China).

Gene-specific RT-qPCR analysis. Total RNA was extracted from cultured primary human chondrocytes using the PureLink RNA mini kit (cat. no. 12183018A; Ambion; Thermo Fisher Scientific, Inc.) and 2-mercaptoethanol (cat. no. 31350010; Thermo Fisher Scientific, Inc.). The quantity of RNA obtained from each sample was measured using a UV spectrophotometer (UV-VIS Spectrophotometer 2600; Shimadzu Corporation, Kyoto, Japan). To obtain cDNA, 50 ng RNA was reverse transcribed using a high-capacity cDNA reverse transcription kit (cat. no. 4368814; Thermo Fisher Scientific, Inc.) and a thermal cycler (ProFlex; Thermo Fisher Scientific, Inc.). For RT, $10 \mu 1$ $10 \mathrm{X}$ reverse transcription buffer, $4 \mu \mathrm{l} 25 \mathrm{X}$ dNTP, $10 \mu 110 \mathrm{X}$ random primers, $5 \mu 150 \mathrm{U} / \mu 1$ reverse transcriptase, $51 \mu 1$ nuclease free water and $20 \mu \mathrm{l}$ RNA were mixed well and held at $25^{\circ} \mathrm{C}$ for $10 \mathrm{~min}$, and then maintained at $37^{\circ} \mathrm{C}$ for $2 \mathrm{~h}$. All genes were amplified using TaqMan ${ }^{\circledR}$ Gene Expression assays for CHAD (assay ID. Hs00154382_m1; cat. no. 4448892), HIF-1 $\alpha$ (assay ID. Hs00153153_m1; cat. no. 4453320), COL2A1 (assay ID. Hs00264051_m1; cat. no. 4453320) and internal control gene [housekeeping genes-actin beta (ACTB; assay ID. Hs99999903_m1; cat. no. 4453320)]. qPCR was performed on an Applied Biosystems 7300/7500 real-time PCR system (Thermo Fisher Scientific, Inc.) and the reaction mixture consisted of $1 \mu 1$ TaqMan gene expression assay, $10 \mu 1$ TaqMan gene expression master mix (cat. no. 4369016), $4 \mu \mathrm{l}$ cDNA template and UltraPure DNase/RNase-free distilled water (cat. no. 10977035) for each gene in MicroAmp fast optical 96-well reaction plates (cat. no. 4346906). The thermocycling conditions were as follows: $2 \mathrm{~min}$ at $50^{\circ} \mathrm{C}, 10 \mathrm{~min}$ at $95^{\circ} \mathrm{C}$, $15 \mathrm{sec}$ at $95^{\circ} \mathrm{C}$ and $1 \mathrm{~min}$ at $60^{\circ} \mathrm{C}$, for 40 cycles. As a result of the RT-qPCR experiment, the RQ values of each sample were obtained using the 7500 Fast-SDS program V.2.3 (Thermo Fisher Scientific, Inc.). An endogenous control (ACTB) was utilized to normalize the target gene expression. For comparative results, a reference (calibrator) sample (Group 1, $0 \mathrm{~h}$ ) was used and relative quantity (RQ) values were calculated using the $2^{-\Delta \Delta \mathrm{Cq}}$ method (21).

Statistical analysis. Statistical analysis was performed using the Minitab program (version 16; Minitab, Inc., State College, PA, USA). All data are expressed as the mean \pm standard deviation. The results were evaluated using repeated measures one-way analysis of variance to assess whether there were significant differences across experimental groups, followed by Tukey's honest significant difference test for post hoc analysis. $\mathrm{P}<0.05$ was considered to indicate a statistically significant difference. All experiments were repeated at least three times. To avoid measurement errors, the same types of analysis were performed by the same researchers.

\section{Results}

Morphological evaluation of chondrocyte cultures. Chondrocyte morphology was evaluated 24 and $48 \mathrm{~h}$ following treatment with low, medium and high MW HA. Cell proliferation in the control group at $24 \mathrm{~h}$ was greater than in all HA-treated experimental 

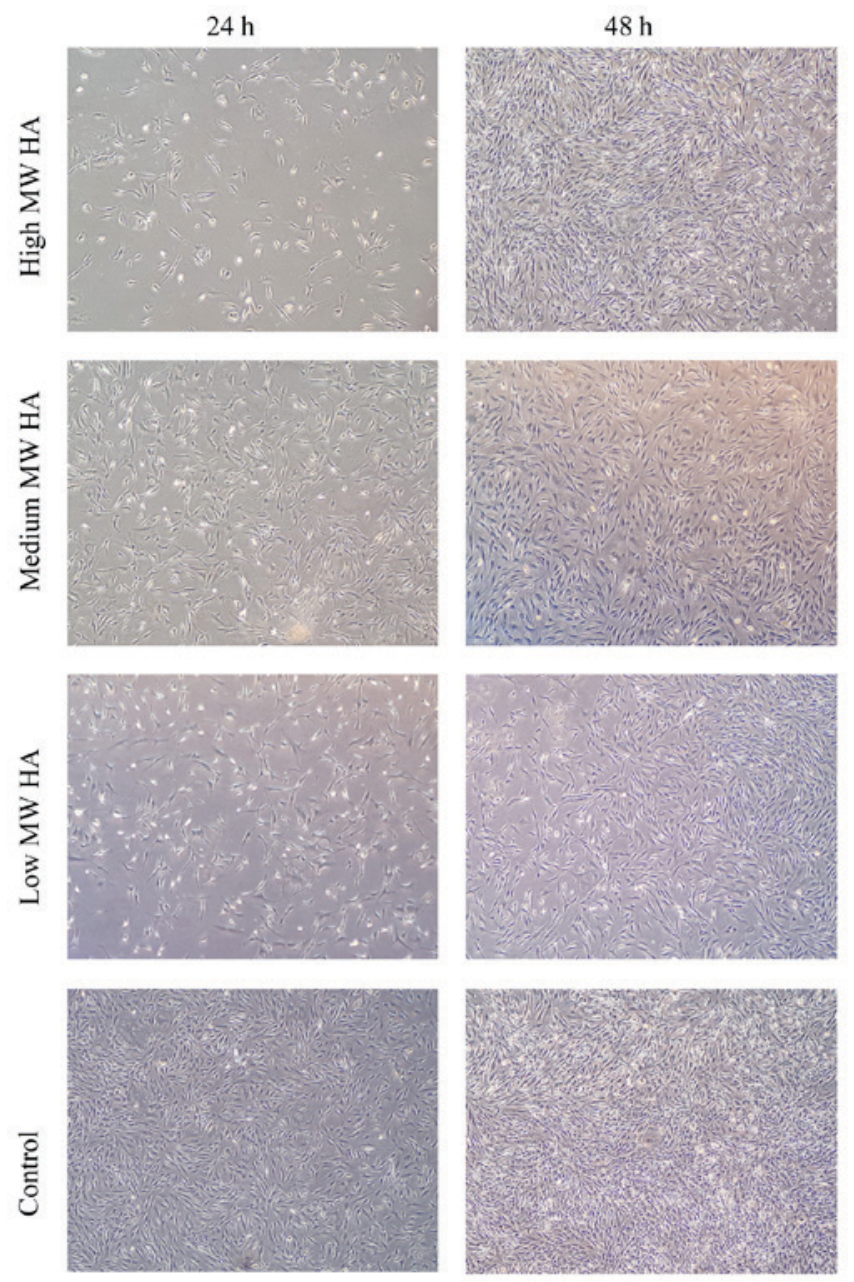

Figure 1. Inverted microscopy images of primary chondrocyte cultures (magnification, x20). MW, molecular weight; HA, hyaluronic acid.

groups. Although the number of cells appeared fewer in number than HA-treated groups in morphological observations, MTT analysis demonstrated that the viable cell ratio remained almost the same at $24 \mathrm{~h}$ in all groups. However, it was observed that chondrocyte proliferation increased in all experimental groups at $48 \mathrm{~h}$ following HA treatment, particularly in the high-MW HA treatment group that underwent morphological evaluation (Fig. 1). Despite cultures in HA-treated groups having a weaker confluency following $24 \mathrm{~h}$, to demonstrate that HA or its components do not suppress chondrocyte proliferation and to confirm MTT data cell viability in the same cultures were also assessed using AO/PI staining. Micrographs obtained following AO/PI staining are presented in Fig. 2. Cells retained their specific morphology, maintained viability and continued to proliferate in all experimental groups according to microscopic examinations, indicating that HA does not have a repressive effect (Fig. 2).

Statistical analysis of chondrocyte proliferation. Cell viability was determined by MTT analysis, confirmed by AO/PI staining and evaluated statistically. The results revealed that the increase in cell proliferation was significantly associated with the duration of application $(\mathrm{P}<0.05$; Fig. 3). However, there were no significant differences between the cell viability in the groups treated with HA that had different MWs (Table I).
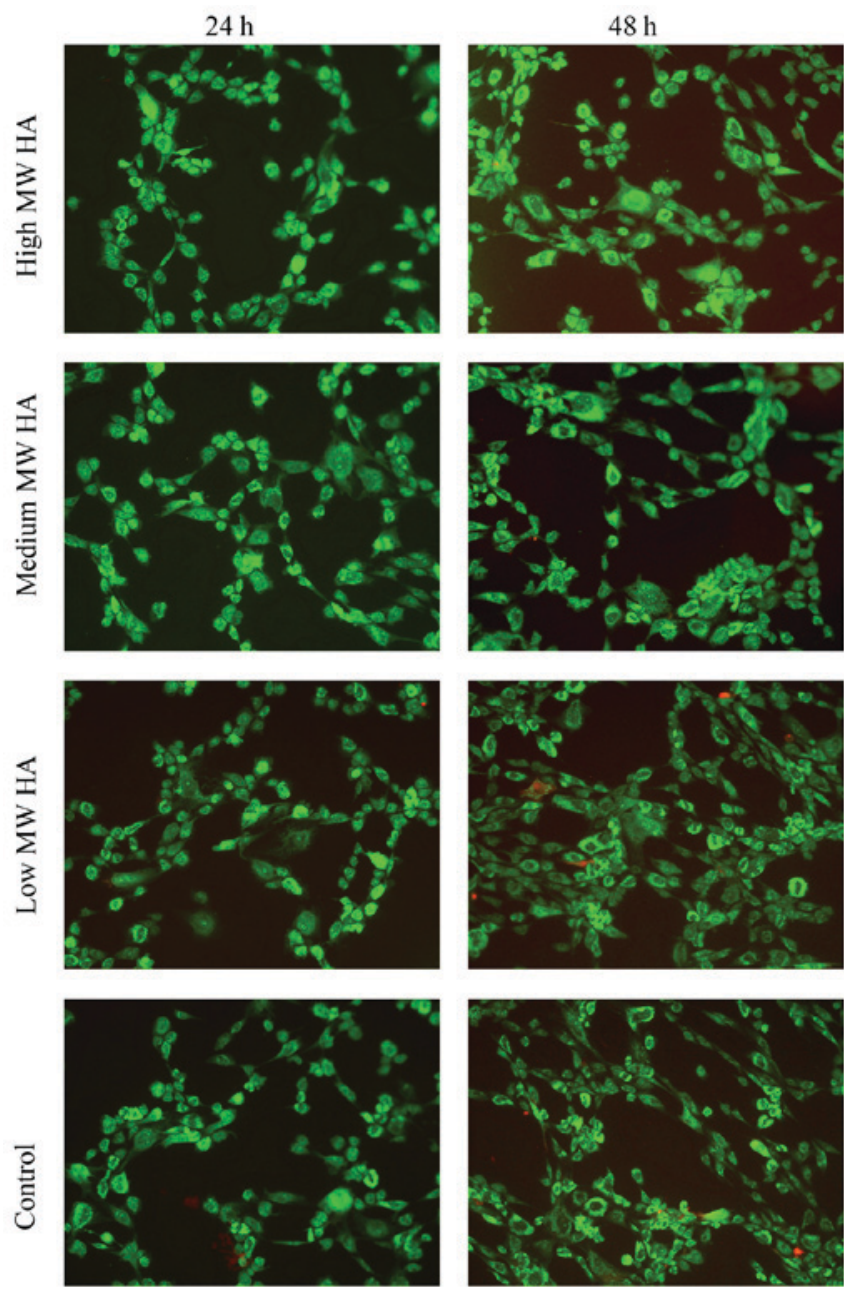

Figure 2. AO/PI staining of chondrocyte cultures. Cells stained green indicate viable chondrocytes. Cells with red nuclei indicate cell death or degenerated membrane integrity (magnification, 20x). MW, molecular weight; HA, hyaluronic acid; $\mathrm{AO}$, acridine orange; $\mathrm{PI}$, propidium iodide.

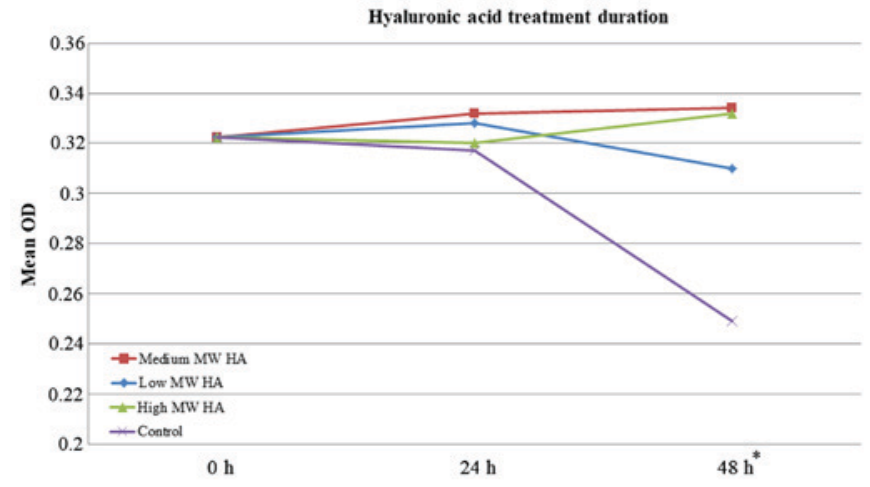

Figure 3. Statistical analyses; pairwise comparisons for MTT analysis assessing cell proliferation. Increased cell viability was statistically significant $\left({ }^{*} \mathrm{P}<0.005\right)$ in all experimental groups (low, medium and high MW HA) when compared to control group at $48 \mathrm{~h}$.

Analysis of CHAD, COL2Al and HIF-l $\alpha$ gene expression. The expression of CHAD,COL2AI and HIF-l $\alpha$ were assessed 24 and $48 \mathrm{~h}$ following treatment with HA at different MWs. RQ) values were calculated for each experimental group using the 7500 Fast-SDS program. $\beta$-actin was used as endogenous 
Table I. Statistical analysis of hyaluronic acid application with one-way analysis of variance and Tukey's post hoc test.

\begin{tabular}{lcccr}
\hline \multirow{2}{*}{$\begin{array}{l}\text { Hyaluronic acid } \\
\text { treatment }\end{array}$} & \multicolumn{3}{c}{ Mean OD \pm SD } \\
\cline { 2 - 4 } & $0 \mathrm{~h}$ & $24 \mathrm{~h}$ & $48 \mathrm{~h}$ & P-value \\
\hline Low MW & $0.322 \pm 0.03$ & $0.328 \pm 0.05$ & $0.310 \pm 0.01$ & 0.310 \\
Medium MW & $0.322 \pm 0.03$ & $0.332 \pm 0.04$ & $0.334 \pm 0.01$ & 0.311 \\
High MW & $0.322 \pm 0.03$ & $0.320 \pm 0.06$ & $0.332 \pm 0.03$ & 0.298 \\
Control & $0.322 \pm 0.03$ & $0.317 \pm 0.06$ & $0.249 \pm 0.01$ & 0.296 \\
\hline
\end{tabular}

Results are presented as the mean \pm standard deviation. MW, molecular weight, OD, optical density, SD, standard deviation. There were no significant differences between the cell viability in the groups treated with HA that had different MWs.

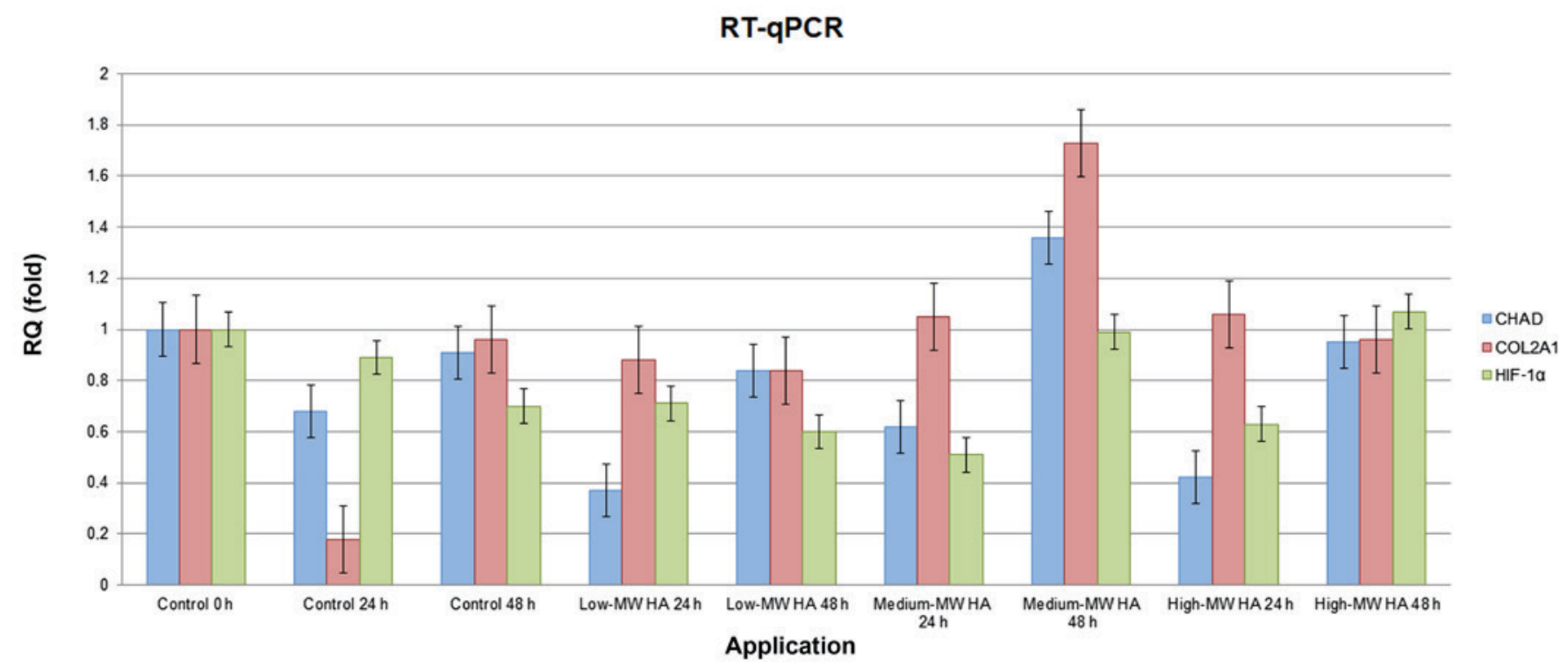

Figure 4. RQ values obtained for chondroadherin, collagen type II $\alpha$ chain 1 and hypoxia-inducible factor $1 \alpha$ expression. HA, hyaluronic acid; MW, molecular weight; RQ, relative quantity.

control and the control group at $0 \mathrm{~h}$ was designated to be the reference sample. The expression of the gene of interest was determined as $100 \%$ and the RQ value was 1 in the reference sample and RQ values of other samples were calculated as fold changes with comparison to this reference (21). No further statistical analysis was performed on the obtained RQ values.

Fig. 4 summarizes the RQ values determined for each experimental group. In the medium MW HA treated group, COL2A1 gene expression increased 1.73-fold and CHAD gene expression increased 1.35 -fold at $48 \mathrm{~h}$ compared with the control group. Furthermore, at $24 \mathrm{~h}$, the COL2A1 gene RQ value was 1.05 . In the high MW HA treated group, the RQ value determined for the COL2A1 gene was 1.06 at $24 \mathrm{~h}$ and RQ value determined for the HIF-1 $\alpha$ gene was 1.07 at $48 \mathrm{~h}$ (Fig. 4).

\section{Discussion}

The primary goal of treatments for cartilage damage is to completely restore hyaline cartilage. Surgery and other treatments are being developed for this purpose. Although there are a number of promising methods and some encouraging clinical results, no treatments have achieved complete restoration $(1,4)$. Viscosupplementation with HA is an example of a promising treatment method. HA injections reduce pain and recover joint function by functioning as a lubricant and shock absorber $(4,6)$. Although not fully understood, HA injections have been demonstrated to inhibit inflammation, decrease cartilage degradation and induce ECM synthesis $(1,6)$. Surgeons cannot yet achieve biological repair following cartilage damage; however, treatment with HA is preferred as it inhibits the pathophysiological pathway leading to arthrosis and reduces the pain and movement limitation experienced by patients (1).

HA concentrations are decreased in the affected joints of patients with OA. It has been suggested that HA treatment is more beneficial than viscous fluid replacement therapy, as it stimulates tissue organization and modulates inflammation and macrophages $(8,19)$. Consequently, various commercial HA preparations with different MWs are clinically used (9,23-25). Certain studies have indicated that high MW HA is more effective than low MW HA in treating OA; however, other studies suggest that there is no substantial evidence to support this (1,24-27). In the present study, chondrocyte cultures were 
morphologically evaluated and cell viability and proliferation were determined. The results revealed that the positive effect of HA treatment on cell proliferation was independent of the MW of HA.

Animal tissue or commercial cell lines were not used in the current study; instead, cartilage tissues were obtained from patients with knee prosthesis during the course of routine clinical practice. Following knee prosthesis, undamaged chondral tissues of the resectioned articular surface were used to prepare primary chondrocyte cultures. Human primary articular chondrocyte cultures model complete tissue as they contain all cell types and ECM components of their original tissue (28-31); therefore the results of the present study may enhance understanding of how cartilage degeneration occurs. However, as the effects of HA in the present study were only evaluated in vitro, the results cannot be generalized to predict the effects of HA in vivo.

In the present study, the effect of HA with different MWs on the levels of COL2A1, HIF- $1 \alpha$ and CHAD expression was investigated. The COL2A1 gene encodes an important cartilage-specific extracellular matrix protein that is synthesized from proliferative chondrocytes and is essential for chondrogenesis $(12,32)$. In the developing cartilage of the growing bone, chondrocytes alter their morphology to become hypertrophic and do not express COL2A1. In adult articular cartilage, hypertrophic differentiation processes do not occur and COL2A1 is expressed (32). However, in OA degenerative articular cartilage, phenotypic conversion to hypertrophic chondrocytes has been reported (32). Furthermore, anabolic and catabolic processes are broadly affected in OA (12) and in cartilage, COL2A1 expression is a marker of anabolic processes (5). A previous study revealed that COL $2 \mathrm{~A} 1$ expression increases when chondrocytes are cultured with HA and growth factors (12). It has also been reported that COL2A1 expression increases in human OA-derived chondrocytes following treatment with high MW HA (5). The results of the present study demonstrated that COL2A1 gene expression increased in chondrocytes following treatment with medium and high MW HA. COL2A1 expression was 5.58-fold higher in the medium MW HA treated group $(\mathrm{RQ}=1.05)$ compared with the control group $(\mathrm{RQ}=0.18)$ at $24 \mathrm{~h}$. Furthermore, COL2A1 expression was 1.8-fold higher in the medium MW HA treated group $(R Q=1.73)$ compared with the control group $(\mathrm{RQ}=0.96)$ at $48 \mathrm{~h}$. A 5.88-fold increase in COL2A1 expression was only observed $(\mathrm{RQ}=1.06)$ in the high MW HA treated group at $24 \mathrm{~h}$. However, COL2A1 expression was the same as the control following $48 \mathrm{~h}$. It was also demonstrated that increased duration of medium MW HA treatment increased the expression of the COL2A1 gene, which is associated with chondrocyte proliferation and ECM formation.

Chondrogenesis is regulated by numerous mechanisms, including growth factors, cytokines and oxygen supply (15). During the initial stages of chondrogenesis, the developing cartilaginous template is avascularized and forms hypoxic niches $(13,15)$. HIF-1 $\alpha$ is a basic helix-loop-helix transcription factor expressed in hypoxic conditions $(13,14)$. HIF-1 is a heterodimer of $\alpha$ and $\beta$ subunits. The expression of the $\alpha$ subunit is induced by hypoxia, whereas the $\beta$ subunit is constitutively expressed in the nucleus. In hypoxic conditions, the activity of the HIF-targeting prolyl hydroxylase enzymes (PHD)1, PHD2 and PHD3 is inactivated; as a result, HIF-1 $\alpha$, is not hydroxylated and accumulates in the cytoplasm. Following this, HIF-1 translocates into the nucleus and dimerizes with HIF-1 $\beta$ to form the HIF-1 complex. This complex subsequently binds to hypoxia responsive elements within the promoter region of target genes, including Sox9, vascular endothelial growth factor A, glycolytic enzymes and glucose transporters, and induces their transcription, stimulating chondrocyte differentiation and hypoxic condition adaptation $(13,15)$. HIF-1 therefore positively regulates cartilage development and regeneration $(13,14)$. Several studies have suggested that HIF-1 $\alpha$ serves a role in regulating hypoxia-induced ECM synthesis in chondrocytes (13-15). In the present study, it was observed that HIF-1 $\alpha$ expression $(\mathrm{RQ}=1.07)$ was 1.53 -fold higher than the control group $(\mathrm{RQ}=0.70)$ at $48 \mathrm{~h}$ in the high $\mathrm{MW} \mathrm{HA}$ group only.

CHAD is a non-canonical class IV small leucine-rich proteoglycan/protein. This $38 \mathrm{kDa}$ protein mediates signaling between chondrocytes and the ECM via $\alpha 2 \beta 1$ integrin, cell surface proteoglycans and type II collagen binding (16). It has been demonstrated that the expression of CHAD is upregulated in chondrocyte cultures (33). CHAD predominantly regulates the formation of the collagen fibrillar network during early skeletal development. Therefore, it has been hypothesized that CHAD downregulation serves a critical role in the initiation and progression of OA (16). In the present study, a 1.49-fold increase in CHAD expression $(\mathrm{RQ}=1.36$ ) following $48 \mathrm{~h}$ was observed in the medium MW group alone.

The present study was performed in in vitro primary cultures that directly reflected the entire structure of the original tissue, increasing the value of the obtained data. However, these primary cultures were established using samples taken from only 6 patients; therefore, further studies are required to definitively establish the effect of different MWs of HA on the expression of different genes in OA. OA is a multifactorial disease; therefore gene expression in different patients may vary due to individual differences, independent from disease (34). In the present study, gene expression increased as the duration of treatment increased in the medium and high MW groups. Furthermore, the increased duration of treatment increased chondrocyte viability and proliferation. Taken together, these results indicate that administration of medium and high MW HA may be successful at treating patients with OA.

In conclusion, the present study evaluated the effects of low, medium and high MW HA on the expression of COL2A1, CHAD and HIF-1 $\alpha$ in a robust in vitro culture of human-derived chondrocytes. Similar to previous studies, an increase in COL2A1, CHAD and HIF-1 $\alpha$ expression and proliferation was observed, particularly in cultures treated with medium and high MW HA. Further studies are required to confirm these results in vivo. Typically, it is difficult to imitate the full scope of OA with all the involved cellular constituents in vitro. Therefore, the majority of in vitro OA models are designed to focus on the late stages of disease, or use cell lines. However, this has significant limitations and it is critical to evaluate the mechanisms that are affected during the early 
stages of OA and in primary cell cultures. The present study demonstrated that HA administration at different molecular weights to cell cultures, over short periods (24 and $48 \mathrm{~h}$ ) changed the expression of certain genes. Further study into gene expression is required to define treatment protocols and elucidate the pathogenesis of OA. However, clinicians should consider the cellular effects of HA molecular weight in the treatment of OA.

\section{Acknowledgements}

Not applicable.

\section{Funding}

No funding was received.

\section{Availability of data and materials}

The analyzed data sets generated during the present study are available from the corresponding author on reasonable request.

\section{Authors' contributions}

DYS contributed to the study concept and design and preparation of manuscript, as well as the acquisition of subjects and data, analysis and interpretation of data, preparation of human primer chondrocyte culture, and performance and evaluation of PCR analyses. NK contributed to the study concept and design, acquisition of subjects and data, and the writing of the discussion section. IY contributed to the preparation of human primer chondrocyte culture, inverted light microscopy, performance and evaluation of ELISA analyses, as well as the preparation and storage of culture drugs. IY and HO contributed to the statistical evaluation of the findings and writing of the manuscript. YA selected the patients who met the inclusion criteria and contributed to the statistical evaluation of findings. YK, OG, KO, SA and MM diagnosed and operated on patients, and contributed to the removal of tissues from the cases and their transfer to the laboratory. HO, NK, NA, DYS contributed to the preparation and critical revision of the manuscript for important intellectual content. NA also prepared the drugs and adapted the clinical doses to cell cultures. All authors have read and approved the final version of the manuscript.

\section{Ethics approval and consent to participate}

This study was performed with the approval of the Local Ethics Board of Pamukkale University (Denizli, Turkey) and informed consent was obtained from all patients for use of their cells.

\section{Consent for publication}

Not applicable.

\section{Competing interests}

The authors declare that they have no competing interests.

\section{References}

1. Aggarwal A and Sempowski IP: Hyaluronic acid injections for knee osteoarthritis. Systematic review of the literature. Can Fam Physician 50: 249-256, 2004

2. Zelzer E, Mamluk R, Ferrara N, Johnson RS, Schipani E and Olsen BR: VEGFA is necessary for chondrocyte survival during bone development. Development 131: 2161-2171, 2004.

3. Charlier E, Relic B, Deroyer C, Malaise O, Neuville S, Collée J, Malaise MG and De Seny D: Insights on molecular mechanisms of chondrocytes death in osteoarthritis. Int J Mol Sci 17: E2146, 2016.

4. Neustadt DH: Intra-articular injections for osteoarthritis of the knee. Cleve Clin J Med 73: 897-898, 901-904, 906-911, 2006.

5. Bauer C, Niculescu-Morzsa E, Jeyakumar V, Kern D, Späth SS and Nehrer S: Chondroprotective effect of high-molecular-weight hyaluronic acid on osteoarthritic chondrocytes in a co-cultivation inflammation model with M1 macrophages. J Inflamm (Lond) 13: 31, 2016.

6. Kelly MA, Moskowitz RW and Lieberman JR: Hyaluronan therapy: Looking toward the future. Am J Orthop (Belle Mead NJ) 33 (2 Suppl): S23-S28, 2004.

7. McGrath AF, McGrath AM, Jessop MA, Gandham S, Datta G, Dawson-Bowling S and Cannon SR: A comparison of intra-articular hyaluronic acid competitors in the treatment of mild to moderate knee osteoarthritis. J Arthritis 2: 1, 2013.

8. Kelly MA, Kurzweil PR and Moskowitz RW: Intra-articular hyaluronans in knee osteoarthritis: Rationale and practical considerations. Am J Orthop (Belle Mead NJ) 33 (2 Suppl): S15-S22, 2004.

9. Ghosh P and Guidolin D: Potential mechanism of action of intra-articular hyaluronan therapy in osteoarthritis: Are the effects molecular weight dependent? Semin Arthritis Rheum 32: 10-37, 2002.

10. Barreto RB, Sadigursky D, de Rezende MU and Hernandez AJ: Effect of hyaluronic acid on chondrocyte apoptosis. Acta Ortop Bras 23: 90-93, 2015.

11. Patti AM, Gabriele A, Vulcano A, Ramieri MT and Della Rocca C: Effect of hyaluronic acid on human chondrocyte cell lines from articular cartilage. Tissue Cell 33: 294-300, 2001.

12. Klangjorhor J, Phitak T, Pruksakorn D, Pothacharoen P and Kongtawelert P: Comparison of growth factor adsorbed scaffold and conventional scaffold with growth factor supplemented media for primary human articular chondrocyte 3D culture. BMC Biotechnol 14: 108, 2014.

13. Niebler S, Angele P, Kujat R and Bosserhoff AK: Hypoxiainducible factor 1 is an inductor of transcription factor activating protein 2 epsilon expression during chondrogenic differentiation. Biomed Res Int 2015: 380590, 2015.

14. Kozhemyakina E, Lassar AB and Zelzer E: A pathway to bone: signaling molecules and transcription factors involved in chondrocyte development and maturation. Development 142: 817-831, 2015.

15. Wang P, Zhang F, He Q, Wang J, Shiu HT, Shu Y, Tsang WP, Liang S, Zhao K and Wan C: Flavonoid compound icariin activates hypoxia inducible factor- $1 \alpha$ in chondrocytes and promotes articular cartilage repair. PLoS One 11: e0148372, 2016.

16. Batista MA, Nia HT, Önnerfjord P, Cox KA, Ortiz C, Grodzinsky AJ, Heinegård D and Han L: Nanomechanical phenotype of chondroadherin-null murine articular cartilage. Matrix Biol 38: 84-90, 2014.

17. Kellgren JH and Lawrence JS: Radiological assessment of rheumatoid arthritis. Ann Rheum Dis 16: 485-493, 1957.

18. Isyar M, Yilmaz I, Yasar Sirin D, Yalcin S, Guler O and Mahirogullari M: A practical way to prepare primer human chondrocyte culture. J Orthop 13: 162-167, 2016.

19. Rayahin JE, Buhrman JS, Zhang Y, Koh TJ and Gemeinhart RA: High and low molecular weight hyaluronic acid differentially influence macrophage activation. ACS Biomater Sci Eng 1: 481-493, 2015.

20. Mascotti K, McCullough J and Burger SR: HPC viability measurement: Trypan blue versus acridine orange and propidium iodide. Transfusion 40: 693-696, 2000.

21. Livak KJ and Schmittgen TD: Analysis of relative gene expression data using real-time quantitative PCR and the 2(-Delta Delta C(T)) method. Methods 25: 402-408, 2001.

22. VanGuilder HD, Vrana KE and Freeman WM: Twenty-five years of quantitative PCR for gene expression analysis. Biotechniques 44: 619-626, 2008. 
23. Smith MM and Ghosh P: The synthesis of hyaluronic acid by human synovial fibroblasts is influenced by the nature of the hyaluronate in the extracellular environment. Rheumatol Int 7: 113-122, 1987.

24. Aviad AD and Houpt JB: The molecular weight of therapeutic hyaluronan (sodium hyaluronate): How significant is it? J Rheumatol 21: 297-301, 1994.

25. Arrich J, Piribauer F, Mad P, Schmid D, Klaushofer K and Müllner M: Intra-articular hyaluronic acid for the treatment of osteoarthritis of the knee: Systematic review and meta-analysis. CMAJ 172: 1039-1043, 2005.

26. Zhao H, Liu H, Liang X, Li Y, Wang J and Liu C: Hylan G-F 20 vs. low molecular weight hyaluronic acids for knee osteoarthritis: A meta-analysis. Bio Drugs 30: 387-396, 2016.

27. Lo GH, LaValley M, McAlindon T and Felson DT: Intra-articular hyaluronic acid in treatment of knee osteoarthritis: A meta-analysis. JAMA 290: 3115-3121, 2003.

28. Gokce A, Yilmaz I, Bircan R, Tonbul M, Gokay NS and Gokce C: Synergistic effect of TGF- $\beta 1$ and BMP-7 on chondrogenesis and extracellular matrix synthesis: An in vitro study. Open Orthop J 6: 406-413, 2012.

29. Gökçe A, Yılmaz I, Gökay NS, Can L and Gökçe C: Does insulin, transferrin and selenous acid preparation effect chondrocyte proliferation? Acta Orthop Traumatol Turc 48: 313-319, 2014

30. Isyar M, Bilir B, Yilmaz I, Cakmak S, Sirin DY, Guzelant AY and Mahirogullari M: Are biological agents toxic to human chondrocytes and osteocytes? J Orthop Surg Res 10: 118, 2015.
31. Gumustas SA, Yilmaz İ, Isyar M, Sirin DY, Batmaz AG, Ugras AA, Oznam K, Ciftci Zand Mahirogullari M: Assessing the negative impact of phenyl alkanoic acid derivative, a frequently prescribed drug for the suppression of pain and inflammation, on the differentiation and proliferation of chondrocytes. J Orthop Surg Res 11: 70, 2016.

32. Ushijima T, Okazaki K, Tsushima $H$ and Iwamoto Y: CCAAT/enhancer-binding protein $\beta$ regulates the repression of type II collagen expression during the differentiation from proliferative to hypertrophic chondrocytes. J Biol Chem 289: 2852-2863, 2014.

33. Ezura Y, Sekiya I, Koga H, Muneta T and Noda M: Methylation status of $\mathrm{CpG}$ islands in the promoter regions of signature genes during chondrogenesis of human synovium-derived mesenchymal stem cells. Arthritis Rheum 60: 1416-1426, 2009.

34. Pombo-Suarez M, Calaza M, Gomez-Reino JJ and Gonzalez A: Reference genes for normalization of gene expression studies in human osteoarthritic articular cartilage. BMC Mol Biol 9: 17, 2008.

This work is licensed under a Creative Commons Attribution-NonCommercial-NoDerivatives 4.0 International (CC BY-NC-ND 4.0) License. 